\title{
Analisis de los residuales en el calculo de velocidades geocentricas a partir de series de tiempo diarias PPP
}

\author{
Jorge Moya Zamora \\ Sara Bastos Gutiérrez
}

\section{Resumen}

El procesamiento de observaciones GNSS en línea ha venido en aumento en los útlimos años. Diferentes servicios como el ofrecido por el Canadian Space Reference System (CSRS), bajo la modalidad de Posicionamiento Puntal Preciso (PPP), brinda la posibilidad de conocer de una manera rápida y efectiva la posición tridimensional a partir de un archivo rinex. Aprovechando estos resultados, se procesaron archivos diarios por un periodo de 2,5 años y para 10 estaciones GNSS de Costa Rica integradas al Sistema de Referencia Geocéntrico para las Américas (SIRGAS). Las coordenadas geocéntricas derivadas del procesamiento PPP en línea, se consideraron como vectores de observaciones para la estimación de sus velocidades (Moya et al, 2017). No obstante, previamente se programó e implementó una prueba estadística denominada test de errores groseros (Pelzer, 1985) para la estimación de potenciales observaciones atípicas. Esta prueba es usada frecuentemente en el análisis de redes geodésicas (Knight et al, 2010), sin embargo, ahora se aplicó en el modelo lineal de cálculo de velocidades. El proceso de ajuste para el cálculo de las velociadades se hizo de manera iterativa por estación y por coordenada, excluyendo las observaciones groseras marcadas por el test en cada proceso. En promedio esta prueba detectó una serie de observaciones con residuos que variaron entre los $-50 \mathrm{~mm}$ y los $40 \mathrm{~mm}$ en sus tres componentes. Finalmente con las velocidades depuradas de errores groseros se validaron respecto a la solución multianual SIR15P01 de SIRGAS, tomando como a la época de referencia 2017,0 . Las diferencias promedio entre ambas determinaciones fueron de $\pm 15 \mathrm{~mm}$.

Palabras Clave: Test de errores groseros, velocidades, GNSS, SIRGAS

\section{Abstract}

The online processing of GNSS observations has been increasing in recent years. Different services such as the one offered by the Canadian Space Reference System (CSRS), under the Precise Point Positioning (PPP) modality, offers the possibility of knowing in a fast and effective way the three-dimensional position from a rinex file. Taking advantage of these results, daily files were processed for a period of 2.5 years and for 10 GNSS stations in Costa Rica which are integrated into the Sistema de Referencia Geocéntrico para las Américas (SIRGAS). The geocentric coordinates derived from the on-line PPP processing were considered as observation vectors for the estimation of their velocities (Moya et al, 2017). However, a statistical test called the outliers test (Pelzer, 1985) was previously programmed and implemented for the estimation of potential atypical observations. This test is frequently used in the analysis of geodetic networks (Knight 
et al, 2010), however, it was now applied in the linear velocity calculation model. The adjustment process for the calculation of velocities was done iteratively per station and per coordinate, excluding the outlier observations marked by the test in each process. On average, this test detected a series of observations with residuals ranging from $-50 \mathrm{~mm}$ to $40 \mathrm{~mm}$ in its three components. Finally, with the purified velocities of oitliers, they were validated with respect to the SIRGAS multiannual solution SIR15P01, taking as reference time 2017.0. The average differences between the two determinations were $\pm 15 \mathrm{~mm}$.

Keywords: Outliers test, velocity, GNSS, SIRGAS

Jorge Moya Zamora

Costa Rica, Escuela de Ingeniería Topográfica, Centro Nacional de Procesamiento de natoc jorge.moya.zamora@una.cr

Sara Bastos Gutiérrez

Costa Rica, Escuela de Ingeniería Topográfica, Centro Nacional de Procesamiento de Datos GNSS. Universidad de Costa Rica, M.Sc. Ing. email: sara.bastos.gutierrez@una.cr

Fecha de Recepción: 19 de octubre de 2018 / Fecha de Aprobación: 16 de noviembre de 2018 


\section{INTRODUCCIÓN}

El cálculo de la variabilidad temporal de una estación GNSS se determina considerando como observaciones sus propias coordenadas geocéntricas. Por ejemplo, en el cálculo de las velocidades de las estaciones de operación continua GNSS de la red SIRGAS-CON, se tiene como principal insumo las coordenadas derivadas del procesamiento científico semanal. Este tipo de procesamiento reúne una serie de modelos y algoritmos que garantizan una alta exactitud en las coordenadas de las estaciones (SIRGAS (2018)).

Actualmente existen diversas posibilidades en línea que brindan resultados de coordenadas de forma sumamente rápida. Estos resultados, aunque de momento no alcanzan la exactitud obtenida por métodos más rigurosos, son altamente confiables y utilizables debido en gran parte a la eficiencia de los diferentes algoritmos y modelos utilizados, así como en la calidad de los parámetros de una serie archivos adicionales usados en el cálculo.

Aprovechando la rapidez y acceso libre a estos servicios, se presentan los resultados que se obtuvieron al considerar como vectores de observaciones originales, las coordenadas geocéntricas calculadas con el Canadian Space Reference System (CSRS) en la modalidad de Posicionamiento de Punto Preciso (PPP) como insumo para el cálculo de velocidades geocéntricas de un grupo de estaciones GNSS de Costa Rica. El modelo lineal de velocidades fue ampliado para incluir el test de errores groseros, el cual hace una evaluación estadística de los residuales de las observaciones posterior al proceso de ajuste.

\section{2. Principio del ajuste de observaciones}

En el caso de la geodesia, se trabaja con muchas observaciones convencionales o satélites que serán usadas para la determinación de las coordenadas de una serie puntos. Estos conjuntos de puntos son llamados redes geodésicas y generalmente, si se cuenta con una estructura geométrica correctamente diseñada y sobre la cual se tenga control de las mediciones, el cálculo de las coordenadas de los vértices y de otra serie de parámetros se hace por medio de un ajuste de observaciones por mínimos cuadrados.

Si se realizan $n$ observaciones o mediciones en el campo para calcular un total de $u$ incógnitas (coordenadas de los puntos) se puede aplicar un ajuste de observaciones por mínimos cuadrados. El modelo matemático de este algoritmo consta de dos partes fundamentales: el modelo funcional que relaciona las nobservaciones de campo $\mathrm{L}$ con las u-incógnitas o parámetros a determinar $\mathrm{X}$ por medio de una serie de funciones $\mathrm{F}$. Y el modelo estocástico, que toma en cuenta los pesos de las observaciones dispuestos en la matriz P (Pelzer (1985)), (Niemeier (2002)), (Kuang (1996)) y (Perović (2005)). Matricialmente las dos partes del modelo matemático se presenta en el conjunto de ecuaciones (1).

$$
\left.\begin{array}{c}
\hat{L}=\underset{n, 1}{F}(\underset{u, 1}{\hat{X}}) \\
\underset{n, n}{P}=\underset{n, n}{Q_{l l}^{-1}} ; \quad \sigma_{0}^{2} Q_{n, n}=\sum_{\substack{l l \\
n, n}}
\end{array}\right\}
$$

Donde: 


\section{$\hat{L}:$ Vector de observaciones ajustadas.}

$\hat{X}$ : Vector de incógnitas ajustadas

$\sum_{i j}$ :Matriz de variaza-covarianza de las observaciones originales

$Q_{j j}$ :Matriz de factores y cofactores de las observaciones originales

$\sigma_{0}^{2}$ : Varianza apriori de la unidad de pesos

$\mathrm{P}$ : Matriz de pesos de las observaciones originales

El modelo funcional expresado en (1) es teórico y parte de los valores verdaderos de las incógnitas, sin embargo, para mantener la consistencia en el sistema toman las observaciones ajustadas, las cuales son las observaciones originales a las que se las ha aplicado pequeñas correcciones que siguen el principio de mínimos cuadrados. Además, las funciones consideradas en el vector $\mathrm{F}$ relacionan las observaciones de campo con las incógnitas. Estas funciones deben linealizarse, para lo cual se parte de un valor aproximado para las incógnitas 0

${ }_{x}^{0}$ permitiendo descartar los términos de segundo orden en la linealización. Los coeficientes respectivos se agrupan en la matriz de configuración o matriz de diseño A como se expresa en (2).

$$
A=u=\left[\begin{array}{ccccc}
\frac{\partial F_{1}}{X_{1}} & \frac{\partial F_{1}}{X_{2}} & \frac{\partial F_{1}}{X_{3}} & \ldots & \frac{\partial F_{1}}{X_{u}} \\
\frac{\partial F_{2}}{X_{1}} & \frac{\partial F_{2}}{X_{2}} & \frac{\partial F_{2}}{X_{3}} & \ldots & \frac{\partial F_{2}}{X_{u}} \\
\frac{\partial F_{3}}{X_{1}} & \frac{\partial F_{3}}{X_{2}} & \frac{\partial F_{3}}{X_{3}} & \ldots & \frac{\partial F_{3}}{X_{u}} \\
\vdots & \vdots & \vdots & \vdots & \vdots \\
\frac{\partial F_{n}}{X_{1}} & \frac{\partial F_{n}}{X_{2}} & \frac{\partial F_{n}}{X_{3}} & \ldots & \frac{\partial F_{n}}{X_{u}}
\end{array}\right]
$$

Finalmente se introduce un vector de observaciones reducidas I y el vector de incógnitas reducidas ajustadas $\hat{x}$ que junto con el vector de residuos o correcciones $v$ se plantea el modelo funcional linealizado del ajuste de observaciones como se representa en (3).

$$
\underset{n, l}{l}+\underset{n, l}{v}=\underset{n, u u, 1}{A} \hat{x}
$$

Donde:

$l$ : Vector de observaciones reducidas

$v$ : Vector de residuos

$A$ : Matriz de configuración

$\hat{x}$ : Vector de observaciones reducidas ajustadas

La solución del sistema está basado en el principio de mínimos cuadrados y se representa en el conjunto de ecuaciones (4):

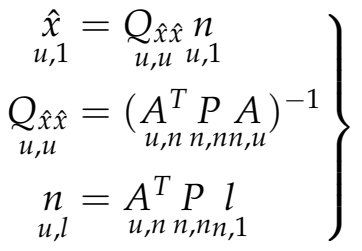

Donde:

$Q_{\hat{X} \hat{x}}:$ Matriz de factores y cofactores de las incógnitas ajustadas 
$n$ : Vector de términos independientes

Conociendo el vector de incógnitas reducidas, se calcula el vector de residuos con (3) y posteriormente los vectores de incógnitas ajustadas y de observaciones ajustadas de la siguiente manera:

$$
\left.\begin{array}{c}
\underset{u, 1}{\hat{X}}=\underset{u, 1}{X}+\underset{u, 1}{\hat{x}} \\
\hat{L}=\underset{n, 1}{L}+\underset{n, 1}{v}
\end{array}\right\}
$$

\section{El test de errores groseros en redes geodésicas}

Se consideran como errores groseros aquellos que se originan en una equivocación, un error en el proceso de medición o en la transmisión o elaboración de datos referentes a las observaciones originales. No son errores de carácter sistemático, ni son errores accidentales, casuales o aleatorios, aunque pueden estar afectados de ese tipo de componente. Los errores groseros afectan todo el conjunto de mediciones, se propagan y eventualmente se diluyen, lo que hace difícil su localización. La teoría de errores excluye a los errores de este tipo, dejando solamente los errores inherentes a los observaciones para su tratamiento.

En el proceso de ajuste de observaciones por mínimos cuadrados, se hace un tratamiento a los residuales de las observaciones para comprobar o descartar la influencia de potenciales errores groseros que afecten los resultados finales, partiendo del principio de que cualquiera las observaciones puede estar afectadas de errores groseros y que solamente una (Pelzer (1985)) y (Niemeier (2002)). El test está diseñado de forma que en caso de localizar varias observaciones atípicas debe ser capaz de encontrar la de mayor error grosero.

La deducción completa del test se puede consultar en Pelzer (1985). Sin embargo, de manera general se parte del modelo funcional linealizado del algoritmo de ajuste de observaciones descrito en (3), el cual se amplía sumando una incógnita adicional llamada $\hat{\Delta} j$ que será precisamente el error grosero que afecta a la observación j como se representa en el conjunto de ecuaciones (6).

$$
\left.\begin{array}{rl}
\hat{\imath} & =l+v=A \hat{x} \\
\hat{\imath} & =L+v=\left[\begin{array}{ll}
A & e_{j}
\end{array}\right]\left[\begin{array}{c}
\hat{x} \\
\hat{\Delta} j
\end{array}\right] \\
e_{j}^{T} & =\left[\begin{array}{llllll}
0 & 0 & \cdots & \cdots & 1 \cdots & 1
\end{array}\right]
\end{array}\right\}
$$

Se plantea la hipótesis nula $H_{0}$ asumiendo que el error $\hat{\Delta} j$ será nulo y la hipótesis alternativa $H_{A}$ en la que error grosero será diferente de cero. A partir de las correspondientes matrices de cofactores de las observaciones originales (1) y de las observaciones ajustadas, se deduce los valores para la j-ésima observación 
como se expresa en el conjunto de ecuaciones (7).

$$
\left.\begin{array}{l}
Q_{v v}=Q_{l l}-Q_{\hat{\imath}} \\
q_{v_{j}}=q_{l_{j}}-q_{\hat{l}_{j}} \\
\vdots \\
q_{v_{j}}=\frac{\sigma_{j}^{2}}{\sigma_{0}^{2}}-\frac{S_{j}^{2}}{S_{0}^{2}}
\end{array}\right\}
$$

Donde:

$Q_{v v}$ : Matriz de cofactores de los residuos v

$Q_{l l}$ : Matriz de factores y cofactores de las observaciones originales

$Q_{\hat{\imath} l}$ : Matriz de cofactores de las observaciones ajustadas

$\sigma_{j}^{2}$ : Varianza teórica de la j-ésima observación

$S_{j}^{2}$ : Varianza empírica de la j-ésima observación

A partir de estos valores se determina el residuo normalizado con la ecuación (8) en función de su desviación estándar normalizada y de los grados de libertad $f$ del ajuste con la ecuación (9). Este resultado será el valor de prueba o estadístico t que se someterá a las hipótesis del test.

$$
\begin{gathered}
\bar{v}_{j}=\frac{\left|V_{j}\right|}{\bar{s}_{0} \sqrt{q_{v_{i}}}}=t \\
\bar{s}_{o}=\sqrt{\frac{1}{f-1}\left(f s_{0}^{2}-\frac{v_{j}^{2}}{q_{v_{i}}}\right)}
\end{gathered}
$$

Finalmente la comparación del valor de prueba o estadístico t se hace con el cuantil de la distribución T-Student. Si el valor del estadístico t es estrictamente mayor que el valor del cuantil, la hipótesis nula se rechazará implicando que la j-ésima observación está afectada de algún elemento que produce una perturbación en el modelo matemático. Si por el contrario, el valor del estadísitico es menor o igual que el cuantil de la distribución, la hipótesis nula se aceptará evidenciando que la observación no está afectada de error estadísticamente. El cuantil se calcula con la relación (10), donde $\square$ el es nivel de incertidumbre dado generalmente para un $5 \%$. El lector no debe confundir el estadístico t con el cuantil de la distribución T-Student denominado también como t.

$$
\left.\begin{array}{l}
t_{f-1,1-\frac{\bar{a}}{2}} \\
\frac{\bar{a}}{2}=1-\sqrt[n]{1-\frac{a}{2}}
\end{array}\right\}
$$

\section{Modelo lineal de velocidades}

Los detalles de la formulación completa del modelo lineal para la estimación de velocidades de la estaciones GNSS a partir de sus coordenadas geocéntricas 
se puede consultar por ejemplo en s. Moya J.and Bastos y Rivas (2014). La realización de los marcos de referencia actualmente está dada por las coordenadas $\mathrm{XO}, \mathrm{Y} 0, \mathrm{ZO}$ para una época fija t0 y sus variaciones lineales en el tiempo dX/dt, $d Y / d t, d Z / d t$ y las coordenadas del vector $X$ de una época ti se extrapolan por medio de la relación (11).

$$
\left.\begin{array}{rl}
X\left(t_{i}\right) & =X_{0}+\frac{d X}{d t}\left(t_{i}-t_{0}\right) \\
X_{0}^{T} & =\left[\begin{array}{lll}
X_{0} & Y_{0} & Z_{0}
\end{array}\right]
\end{array}\right\}
$$

Las velocidades así determinadas siguen un modelo lineal, que no corresponde exactamente con el comportamiento real de muchas de las estaciones, que pueden estar afectadas eventualmente de otras fuentes externas de caracter físico (s. Moya J.and Bastos y Rivas (2014)). El tiempo requerido para la estimación de la velocidad de una estación GNSS de operación continua debe ser de al menos dos años, de manera que se puedan contemplar los posible efectos de caracter cíclico como por ejemplo cargas por lluvia o nieve entre otras.

\section{La red de estaciones GNSS de operación continua}

Se tomaron un total de 10 estaciones GNSS de la red SIRGAS-CON de Costa Rica, las cuales son procesadas semanalmente por tres centro de procesamiento SIRGAS diferentes, siendo uno de ellos, el Centro Nacional de Procesamiento de Datos GNSS (CNPDG) de la Universidad Nacional en Costa Rica. En la figura 1 se presenta la ubicación de las estaciones consideradas. 


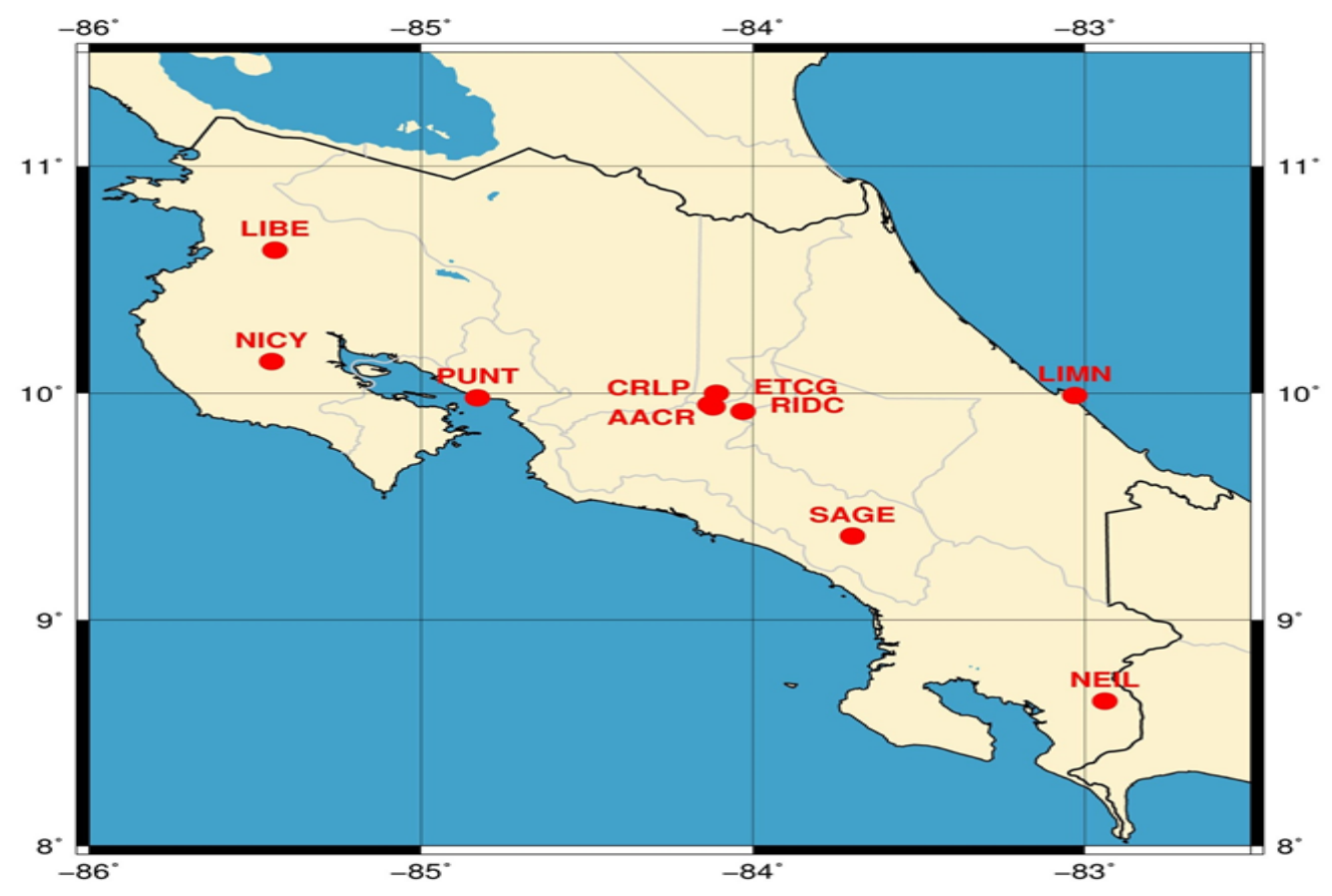

Figura 1: Ubicación de las estaciones GNSS de operación continua de Costa Rica que están integradas a la red SIRGAS-CON

Para la investigación se tomó como un periodo de observación comprendido entre el 27 de julio de 2014 (semana GPS 1803) y el 7 de enero de 2017 (semana GPS 1930). Lo anterior representa un total de 896 días de datos, que corresponden a 127 semanas para 2,44 años. En la tabla 1 se resume la cantidad de archivos procesados por año y por estación.

Tabla 1: Cantidad de días procesados por año para cada una de las estaciones consideradas.

\begin{tabular}{|c|c|c|c|c|c|c|c|c|c|}
\hline EST & $\mathbf{2 0 1 4}$ & $\mathbf{2 0 1 5}$ & $\mathbf{2 0 1 6}$ & $\mathbf{2 0 1 7}$ & EST & $\mathbf{2 0 1 4}$ & $\mathbf{2 0 1 5}$ & $\mathbf{2 0 1 6}$ & $\mathbf{2 0 1 7}$ \\
\hline AACR & 133 & 325 & 354 & 7 & NEIL & 156 & 334 & 260 & 7 \\
\hline CRLP & 0 & 67 & 330 & 7 & NICY & 155 & 362 & 111 & 0 \\
\hline ETCG & 122 & 312 & 337 & 7 & PUNT & 147 & 335 & 318 & 2 \\
\hline LIBE & 157 & 358 & 320 & 7 & RIDC & 156 & 312 & 348 & 7 \\
\hline LIMN & 158 & 359 & 163 & 7 & SAGE & 158 & 347 & 355 & 7 \\
\hline
\end{tabular}

Los archivos de observación de las 10 estaciones de la red SIRGAS de Costa Rica fueron facilitados por el CNPDG. Este laboratorio es el centro oficial de procesamiento para SIRGAS de Costa Rica y realiza la descarga diaria de estos archivos en formato rinex de 24 horas, los cuales fueron previamente editados a registros cada 30 segundos y contemplando mediciones GPS.

El procesamiento de los archivos de manera de obtener las coordenadas geocéntricas se hizo bajo la modalidad PPP (Alves Costa, Silva, Moura, Gende, y 
Brunini (2009)) y (Mireault, Tétreault, Lahaye, Hëroux, y Kouba (2008)) y usando la herramienta CSRS la cual está disponible de manera gratuita en línea en la siguiente dirección de internet:

http://www.nrcan.gc.ca/earth-sciences/geomatics/geodetic-reference-systems/ tools-applications/10925\#ppp La configuración de la herramienta CSRS se hizo siguiendo el mismo procedimiento descrito por Moya et al (2017), es decir usando como referencia geodésica el ITRF y modalidad de procesamiento estático. Luego de que los archivos rinex son procesados por el CSRS, los resultados son enviados vía correo electrónico a una dirección previamente elegida.

\section{Implementación del test de errores groseros}

El modelo lineal de velocidades asumido para la descripción de la cinemática de las estaciones consideradas fue programado en lenguaje de MatLab. Esta rutina calcula la velocidad de una estación GNSS en sus componentes geocéntricas $\left[\begin{array}{lll}X & Y & Z\end{array}\right]^{T}$ a partir de los valores de sus respectivas coordenadas y la época a la cual fueron determinadas. La rutina es denominada como VelMIC (s. Moya J.and Bastos y Rivas (2014)).

Se hizo un ampliación de esta rutina, primeramente haciendo un cálculo individual de las velocidades, es decir por componentes independientes. Esto es válido ya que aunque en el resultado del procesamiento PPP en línea se brinda una estimación de las covarianzas entre los resultados, éstos no se incluyeron en el archivo original de datos. Adicionalmente, se incorporó ahora la posibilidad de elegir el modelo estocástico para las observaciones independientes, es decir, las coordenadas $X, Y$ y Z . Una muestra de la salida de la rutina en su primera corrida se puede ver en la figura 2 donde el algoritmo está aplicado a la coordenada X de la estación AACR y se nota en color rojo al final, el resultado del test de errores groseros. 


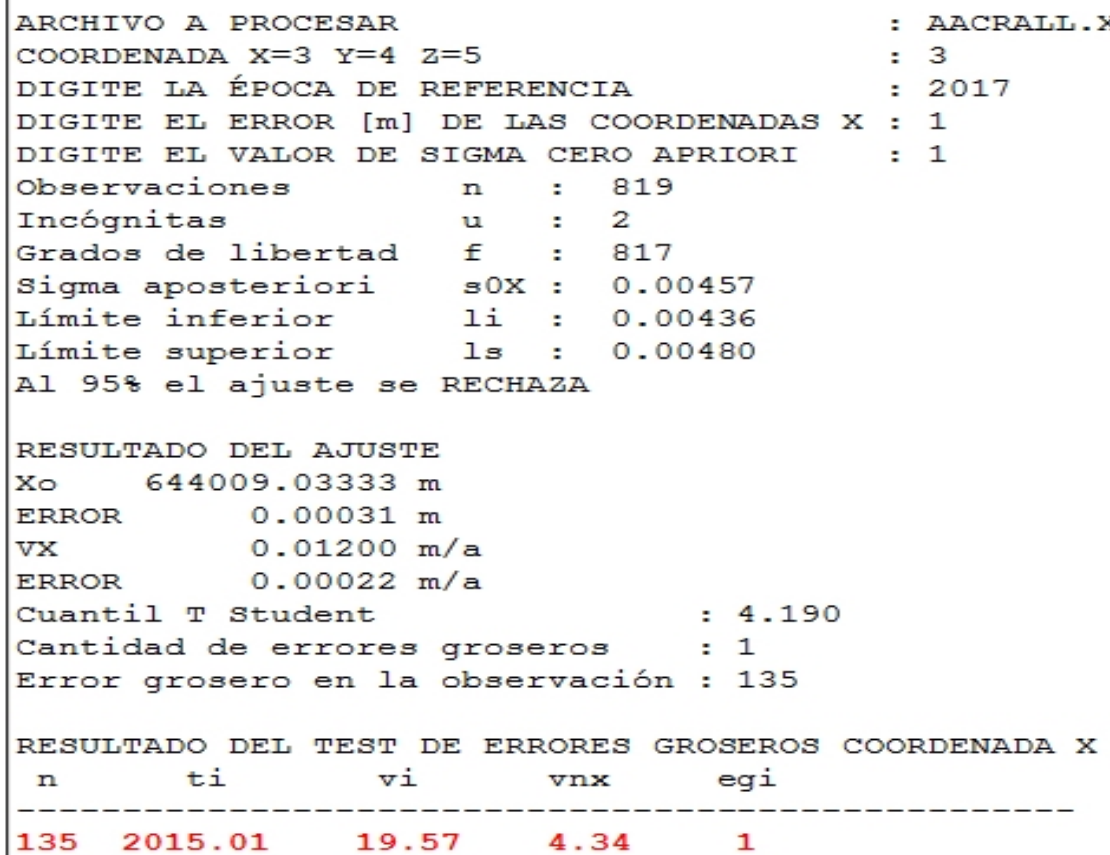

Figura 2: Captura de pantalla de la rutina VelMIC ampliada con el test de errores groseros para la coordenada $X$ de la estación AACR

La metodología aplicada en esta parte consistió en fundamentalmente en los siguientes pasos:

1. Un primer ajuste para la estimación de la velocidad en primer instancia, contemplando una matriz de pesos identidad para las observaciones y los valores del test de errores groseros que demuestran una perturbación en el modelo matemático del ajuste y en este caso en el modelo matemático del ajuste y en este caso en el modelo lineal de velocidades (s. Moya J.and Bastos y Rivas (2014)) y (J. Moya, Núñez, y Cubero (2017)).

2. Posteriormente y dependiendo de cantidad de errores groseros detectados en cada una de la coordenadas de la estación, se hizo un proceso iterativo, en el cual se fueron descartando las observaciones que el test marcó como groseras.

3. Seguidamente, se hizo el posterior equilibrio de varianzas de acuerdo con los resultados del test global del ajuste que no identificara observaciones groseras.

4. Finalmente, el valor asumido como velocidad final para estación fue la obtenida de este ajuste, es decir, el cual está libre de pertubaciones (errores groseros).

\section{RESULTADOS}

En la tabla 2 se resumen la cantidad de datos efectivamente procesados por estación, así como los resultados del ajuste de observaciones directas en [mm] aplicado a las diferencias de coordenadas para cada una de las coordenadas geocéntricas. Las $X$ en las columnas número 3, 6 y 9 corresponden con el valor ajustado, se denominan como $\mathrm{X}$ para estar en concordancia con la nomenclatura clásica. 
Tabla 2: Cantidad de días procesados y resultados del ajuste de observaciones directas aplicado a las diferencias de coordenadas geocéntricas.

\begin{tabular}{|c|c|c|c|c|c|c|c|c|c|c|}
\hline \multirow{2}{*}{ EST. } & \multirow{2}{*}{ Días } & \multicolumn{3}{|c|}{ Coordenada X } & \multicolumn{3}{c|}{ Coordenada Y } & \multicolumn{3}{c|}{ Coordenada Z } \\
\cline { 3 - 11 } & & $X$ & $s_{0}$ & $s_{x}$ & $X$ & $s_{0}$ & $s_{x}$ & $X$ & $s_{0}$ & $s_{x}$ \\
\hline AACR & 819 & 13.29 & 9.72 & 0.34 & -2.20 & 16.44 & 0.57 & 20.08 & 11.75 & 0.41 \\
\hline CRLP & 404 & 7.74 & 7.19 & 0.36 & 7.62 & 8.57 & 0.43 & 14.02 & 7.22 & 0.36 \\
\hline ETCG & 778 & 12.50 & 7.91 & 0.28 & 6.88 & 8.98 & 0.32 & 19.94 & 13.24 & 0.47 \\
\hline LIBE & 842 & 1.71 & 5.54 & 0.19 & 9.66 & 9.27 & 0.32 & 11.06 & 9.44 & 0.33 \\
\hline LIMN & 687 & 16.23 & 9.28 & 0.35 & 12.25 & 11.93 & 0.46 & 2.83 & 7.00 & 0.27 \\
\hline NEIL & 757 & 24.37 & 15.51 & 0.56 & 20.49 & 10.39 & 0.38 & 20.69 & 15.25 & 0.55 \\
\hline NICY & 628 & 2.55 & 5.21 & 0.21 & 4.58 & 7.71 & 0.31 & 6.33 & 7.32 & 0.29 \\
\hline PUNT & 802 & 15.25 & 8.46 & 0.30 & 13.55 & 8.70 & 0.31 & 16.10 & 10.58 & 0.37 \\
\hline RIDC & 823 & 11.85 & 8.75 & 0.30 & 10.01 & 9.64 & 0.34 & 17.51 & 13.47 & 0.47 \\
\hline SAGE & 867 & 26.00 & 16.71 & 0.57 & 5.18 & 9.93 & 0.34 & 26.69 & 17.50 & 0.59 \\
\hline
\end{tabular}

Adicionalmente, se calcularon los intervalos de confianza en [mm] de los valores ajustados cuyos resultados se presentan en la tabla 3. 
Tabla 3: Intervalos de confianza para los valores ajustados de diferencias de coordenadas geocéntricas.

\begin{tabular}{|c|c|c|c|c|c|c|}
\hline \multirow{2}{*}{ EST. } & Límite Inferior & Límite Superior & Límite Inferior & Límite Superior & Límite Inferior & Límite Superior \\
\cline { 2 - 7 } & $\mathrm{a}$ & $\mathrm{b}$ & $\mathrm{a}$ & $\mathrm{b}$ & $\mathrm{a}$ & $\mathrm{b}$ \\
\hline AACR & 12.63 & 13.96 & -3.33 & -1.08 & 19.28 & 20.89 \\
\hline CRLP & 7.04 & 8.46 & 6.79 & 8.46 & 13.32 & 14.73 \\
\hline ETCG & 11.95 & 13.06 & 6.25 & 7.51 & 19.01 & 20.87 \\
\hline LIBE & 1.33 & 2.08 & 9.03 & 10.29 & 10.42 & 11.69 \\
\hline LIMN & 15.54 & 16.93 & 11.36 & 13.15 & 2.31 & 3.35 \\
\hline NEIL & 23.27 & 25.48 & 19.75 & 21.23 & 19.61 & 21.78 \\
\hline NICY & 2.14 & 2.96 & 3.97 & 5.18 & 5.75 & 6.90 \\
\hline PUNT & 14.67 & 15.84 & 12.94 & 14.15 & 15.37 & 16.84 \\
\hline RIDC & 11.25 & 12.44 & 9.35 & 10.67 & 16.59 & 18.43 \\
\hline SAGE & 24.88 & 27.11 & 4.51 & 5.84 & 25.53 & 27.86 \\
\hline
\end{tabular}

El proceso de equilibrio de varianzas se hizo para evaluar adecuadamente los resultados de las componentes de velocidad. En la tabla 4 se presentan los resultados, en la columna Error grosero se cuantifican la totalidad de los errores detectados en cada uno de los ajustes. El valor cero (0) representa que no hubo error grosero. La columna final vi indica es el valor del residuo en la observación correspondiente. 
Tabla 4: Resultados de los procesos de ajuste iterativos con la aplicación del test de errores groseros.

\begin{tabular}{|c|c|c|c|c|c|c|c|c|c|}
\hline \multirow{7}{*}{ AACR } & \multirow{3}{*}{$X$} & 1 & 819 & NO PASA & 1 & 0,00460 & 1 & 135 & +19.57 \\
\hline & & 2 & 818 & NO PASA & 1 & 0,00450 & 0 & & \\
\hline & & 3 & 818 & PASA & 1 & 1,00410 & 0 & & \\
\hline & \multirow{4}{*}{$Z$} & 1 & 819 & NO PASA & 1 & 0,01400 & 0 & & \\
\hline & & 2 & 819 & $\overline{\text { PASA }}$ & 1 & 0,99820 & 0 & & \\
\hline & & 1 & 819 & NO PASA & 1 & 0,00410 & 0 & & \\
\hline & & 2 & 819 & PASA & 1 & 1,03039 & 0 & & \\
\hline \multirow{7}{*}{ CRLP } & \multirow{3}{*}{$x$} & 1 & 404 & NO PASA & 1 & 0,00465 & 1 & 313 & $-18,51$ \\
\hline & & 2 & 403 & NO PASA & 1 & 0,00456 & 0 & & \\
\hline & & 3 & 403 & PASA & 1 & 1,01346 & 0 & & \\
\hline & \multirow{2}{*}{$Y$} & 1 & 404 & NO PASA & 1 & 0,00840 & 0 & & \\
\hline & & 2 & 404 & PASA & 1 & 0,99276 & 0 & & \\
\hline & \multirow{2}{*}{ Z } & 1 & 404 & NO PASA & 1 & 0,00363 & 0 & & \\
\hline & & 2 & 404 & PASA & 1 & 1,00746 & 0 & & \\
\hline \multirow{10}{*}{ ETCG } & \multirow{4}{*}{$X$} & 1 & 778 & NO PASA & 1 & 0,00444 & 2 & 105 & 21,97 \\
\hline & & & & & & & & 314 & $-20,30$ \\
\hline & & 2 & 776 & NO PASA & 1 & 0,00431 & 0 & & \\
\hline & & 3 & 776 & PASA & 1 & 0,95860 & 0 & & \\
\hline & \multirow{4}{*}{$Y$} & 1 & 778 & NO PASA & 1 & 0,00868 & 2 & 122 & $-36,93$ \\
\hline & & & & & & & & 189 & 44,90 \\
\hline & & 2 & 776 & NO PASA & 1 & 0,00844 & 0 & & \\
\hline & & 3 & 776 & PASA & 1 & 0,99241 & 0 & & \\
\hline & \multirow{2}{*}{ Z } & 1 & 778 & NO PASA & 1 & 0,0037 & 0 & & \\
\hline & & 2 & 778 & PASA & 1 & 1,00114 & 0 & & \\
\hline \multirow{10}{*}{ LIBE } & \multirow{3}{*}{$X$} & 1 & 842 & NO PASA & 1 & 0,00463 & 1 & 746 & 25,41 \\
\hline & & 2 & 841 & NO PASA & 1 & 0,00455 & 0 & & \\
\hline & & 3 & 841 & PASA & 1 & 1,01079 & 0 & & \\
\hline & \multirow{3}{*}{$\mathrm{Y}$} & 1 & 842 & NO PASA & 1 & 0,00921 & 1 & 426 & $-39,93$ \\
\hline & & 2 & 841 & NO PASA & 1 & 0,00911 & 0 & & \\
\hline & & 3 & 841 & PASA & 1 & 1,01271 & 0 & & \\
\hline & \multirow{4}{*}{ Z } & 1 & 842 & NO PASA & 1 & 0,00357 & 2 & 388 & $-16,26$ \\
\hline & & & & & & & & 504 & $-17,76$ \\
\hline & & 2 & 840 & NO PASA & 1 & 0,00347 & 0 & & \\
\hline & & 3 & 840 & PASA & 1 & 0,99175 & & & \\
\hline
\end{tabular}




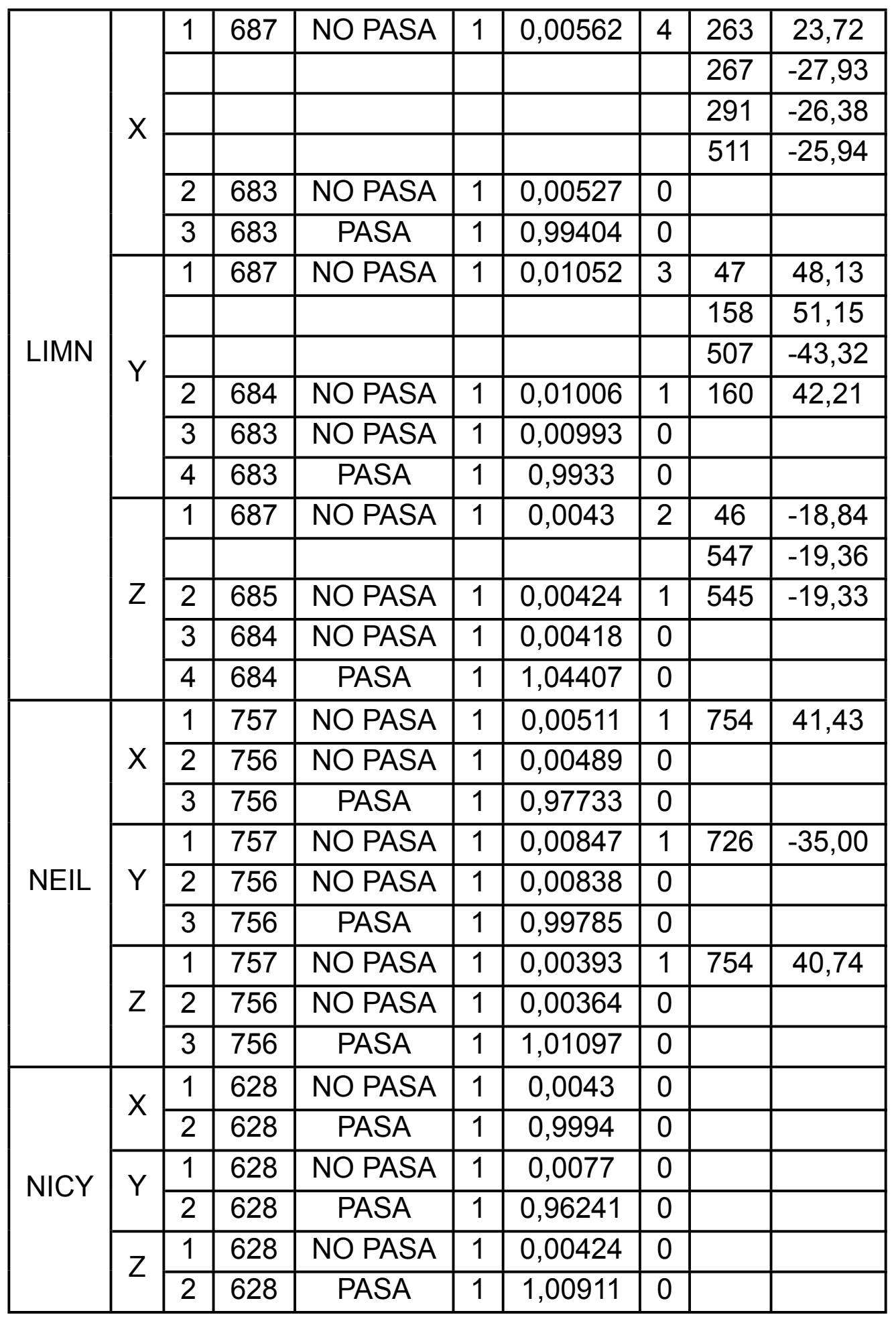




\begin{tabular}{|c|c|c|c|c|c|c|c|c|c|}
\hline \multirow{9}{*}{ PUNT } & \multirow{2}{*}{$X$} & 1 & 802 & NO PASA & 1 & 0,00474 & 0 & & \\
\hline & & 2 & 802 & PASA & 1 & 1,00824 & 0 & & \\
\hline & \multirow{4}{*}{$Y$} & 1 & 802 & NO PASA & 1 & 0,00870 & 2 & 169 & $-47,95$ \\
\hline & & & & & & & & 600 & 36,38 \\
\hline & & 2 & 800 & NO PASA & 1 & 0,00845 & 0 & & \\
\hline & & 3 & 800 & PASA & 1 & 0,99389 & 0 & & \\
\hline & \multirow{3}{*}{ Z } & 1 & 802 & NO PASA & 1 & 0,00366 & 1 & 715 & $-15,41$ \\
\hline & & 2 & 801 & NO PASA & 1 & 0,00362 & 1 & & \\
\hline & & 3 & 801 & PASA & 1 & 1,00587 & 0 & & \\
\hline \multirow{12}{*}{ RIDC } & \multirow{6}{*}{$X$} & 1 & 823 & NO PASA & 1 & 0,0048 & 3 & 121 & 31,48 \\
\hline & & & & & & & & 461 & 20,51 \\
\hline & & & & & & & & 612 & $-23,60$ \\
\hline & & 2 & 820 & NO PASA & 1 & 0,00455 & 1 & 666 & $-18,88$ \\
\hline & & 3 & 819 & NO PASA & 1 & 0,00451 & 1 & & \\
\hline & & 4 & 819 & PASA & 1 & 1,00162 & 1 & & \\
\hline & \multirow{4}{*}{$Y$} & 1 & 823 & NO PASA & 1 & 0,00921 & 1 & 156 & 40,61 \\
\hline & & & & & & & & 808 & 38,83 \\
\hline & & 2 & 821 & NO PASA & 1 & 0,00901 & 0 & & \\
\hline & & 3 & 821 & PASA & 1 & 1,00152 & 0 & & \\
\hline & \multirow{2}{*}{$Z$} & 1 & 823 & NO PASA & 1 & 0,00349 & 0 & & \\
\hline & & 2 & 823 & PASA & 1 & 0,99624 & 0 & & \\
\hline \multirow{8}{*}{ SAGE } & \multirow{3}{*}{$X$} & 1 & 867 & NO PASA & 1 & 0,00505 & 1 & 78 & 22,17 \\
\hline & & 2 & 866 & NO PASA & 1 & 0,00500 & 0 & & \\
\hline & & 3 & 866 & PASA & 1 & 0,99944 & 0 & & \\
\hline & \multirow{3}{*}{$Y$} & 1 & 867 & NO PASA & 1 & 0,00872 & 1 & 362 & $-42,32$ \\
\hline & & 2 & 866 & NO PASA & 1 & 0,00861 & 0 & & \\
\hline & & 3 & 866 & PASA & 1 & 1,00069 & 0 & & \\
\hline & \multirow{2}{*}{ Z } & 1 & 867 & NO PASA & 1 & 0,00341 & 0 & & \\
\hline & & 2 & 867 & PASA & 1 & 1,00157 & 0 & & \\
\hline
\end{tabular}

Luego de la aplicación del test de errores groseros y la adecuación de los pesos de las observaciones, se determinó el valor de las coordenadas y velocidades de referencia, 
Analisis de los residuales en el calculo de velocidades geocentricas a partir de series de tiempo diarias PPP Jorge Moya Zamora Sara Bastos Gutiérrez

estimadas para la época 2017,0, datum ITRF2008 que se presentan en la tabla 5.

Tabla 5: Coordenadas de referencia y velocidades referidas a la época 2017,0, marco ITRF2008 calculadas con base en el procesamiento PPP en línea

\begin{tabular}{|c|c|c|c|c|c|c|}
\hline EST. & \multicolumn{2}{|c|}{ Coordenada(m) } & Error(m) & \multicolumn{2}{|c|}{ Velocidad(m/a) } & Error(m) \\
\hline \multirow{3}{*}{ AACR } & Xo & 644009.03300 & 0.00030 & $\mathrm{VX}$ & 0.01196 & 0.00022 \\
\hline & Yo & -6251064.24790 & 0.00094 & VY & 0.01214 & 0.00068 \\
\hline & Zo & 1093780.92460 & 0.00028 & VZ & 0.01540 & 0.00020 \\
\hline \multirow{3}{*}{ CRLP } & Xo & 643206.60991 & 0.00043 & $\mathrm{VX}$ & 0.01515 & 0.00063 \\
\hline & Yo & -6250876.40150 & 0.00080 & VY & 0.00437 & 0.00120 \\
\hline & Zo & 1095086.10533 & 0.00034 & VZ & 0.01738 & 0.00050 \\
\hline \multirow{3}{*}{ ETCG } & Xo & 645208.32969 & 0.00029 & $\mathrm{VX}$ & 0.00927 & 0.00022 \\
\hline & Yo & -6249842.13405 & 0.00058 & VY & 0.00329 & 0.00043 \\
\hline & Zo & 1100399.63955 & 0.00025 & VZ & 0.01806 & 0.00019 \\
\hline \multirow{3}{*}{ LIBE } & Xo & 498678.63208 & 0.00032 & $\mathrm{VX}$ & 0.00438 & 0.00022 \\
\hline & Yo & -6249679.84934 & 0.00064 & VY & -0.00157 & 0.00044 \\
\hline & Zo & 1168903.06527 & 0.00024 & VZ & 0.01237 & 0.00017 \\
\hline \multirow{3}{*}{ LIMN } & Xo & 762717.32 & 0.00056 & $\mathrm{VX}$ & 0.01295 & 0.00035 \\
\hline & Yo & -6235556.48838 & 0.00106 & VY & 0.00944 & 0.00067 \\
\hline & Zo & 1099500.45327 & 0.00044 & VZ & 0.00975 & 0.00028 \\
\hline \multirow{3}{*}{ NEIL } & Xo & 862 & 0.00039 & $\mathrm{VX}$ & 197 & 0.00027 \\
\hline & Yo & -6258471.53581 & 0.00067 & VY & 0.00885 & 0.00045 \\
\hline & Zo & 952303.07282 & 0.00029 & VZ & 0.02211 & 0.00020 \\
\hline \multirow{3}{*}{$\mathrm{NICY}$} & Xo & 497630.83718 & 0.00056 & $\mathrm{VX}$ & 0.00591 & 0.00034 \\
\hline & Yo & -6259516.91910 & 0.00100 & VY & -0.00108 & 0.00061 \\
\hline & Zo & 1115789.54328 & 0.00055 & VZ & 0.01193 & 0.00034 \\
\hline \multirow{3}{*}{ PUNT } & Xo & 565870.32568 & 0.00034 & $\mathrm{VX}$ & 0.00992 & 0.00024 \\
\hline & Yo & -6256745.20065 & 0.00060 & $\mathrm{VY}$ & -0.00015 & 0.00042 \\
\hline & Zo & 1098060.82868 & 0.00026 & VZ & 0.01403 & 0.00018 \\
\hline \multirow{3}{*}{ RIDC } & Xo & 651566.60857 & 0.00031 & VX & 0.00993 & 0.00022 \\
\hline & Yo & -6250735.94941 & 0.00061 & VY & 0.00395 & 0.00043 \\
\hline & Zo & 1091707.79246 & 0.00024 & VZ & 0.01790 & 0.00017 \\
\hline \multirow{3}{*}{ SAGE } & Xo & 690230.87419 & 0.00034 & VX & 0.02241 & 0.00024 \\
\hline & Yo & -6256292.38755 & 0.00058 & VY & 0.00672 & 0.00041 \\
\hline & Zo & 1032020.69213 & 0.00023 & VZ & 0.02421 & 0.00016 \\
\hline
\end{tabular}


A manera de ejemplo de la salida gráfica de la rutina, se presenta en la figura 3 la serie temporal PPP y la velocidad lineal en las componentes X, Y, Z de las estaciones ETCG y SAGE ubicadas en el sector central y sur del país respectivamente
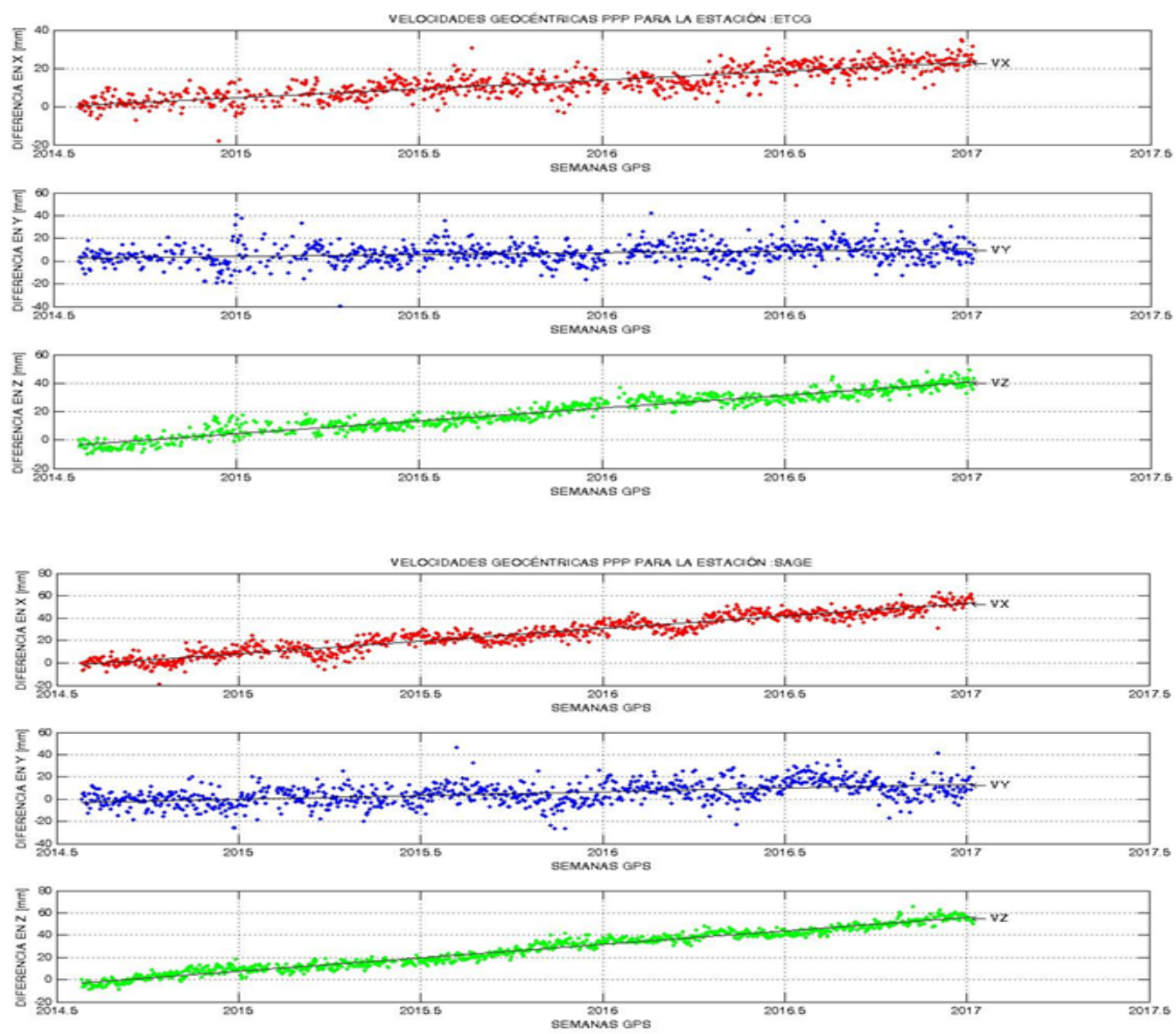

Figura 3: Series temporales PPP. En la parte superior la estación ETCG y en la inferior la estación SAGE

Los resultados en las velocidades se compararon con los datos publicados por el Sistema de Referencia Geocéntrico para las Américas (SIRGAS) en la solución multianual denominada SIR15P01 reducidas a la época 2013,0 (SIRGAS, 2017). En la tabla 6, se presentan los resultados al calcular las coordenadas geocéntricas de las estaciones usando los valores oficiales de SIRGAS extrapolados a la época 2017,0 y determinando las respectivas diferencias de coordenadas. 
Analisis de los residuales en el calculo de velocidades geocentricas a partir de series de tiempo diarias PPP Jorge Moya Zamora Sara Bastos Gutiérrez

Tabla 6: Diferencias de coordenadas geocéntricas producto de la extrapolación de la solución SIR15P01 a la época 2017.0

\begin{tabular}{|c|c|c|c|c|c|}
\hline \multirow[t]{2}{*}{ EST } & \multicolumn{3}{|c|}{ Solución SIR15P01. Época 2013,0 } & \multicolumn{2}{|c|}{ Solución SIR15P01. Época 2017,0 } \\
\hline & \multicolumn{2}{|c|}{ Coordenada(m) } & Velocidad(m/a) & Coordenada(m) & Diferencias(mm) \\
\hline \multirow{3}{*}{ AACR } & $X_{0}$ & 644008.993 & 7.15 & 644009.02185 & 11.2 \\
\hline & $Y_{0}$ & -6251064.284 & 6.97 & -6251064.25575 & 7.9 \\
\hline & Zo & 1093780.865 & 13.94 & 1093780.92086 & 3.7 \\
\hline \multirow{3}{*}{ ETCG } & $X_{0}$ & 645208.293 & 10.56 & 645208.33560 & -5.9 \\
\hline & $Y_{0}$ & -6249842.152 & 4.26 & -6249842.13476 & 0.7 \\
\hline & Zo & 1100399.575 & 17.74 & 1100399.64567 & -6.1 \\
\hline \multirow{3}{*}{ LIBE } & $X_{0}$ & 498678.620 & -0.38 & 498678.61875 & 13.3 \\
\hline & $Y_{0}$ & -6249679.845 & -1.42 & -6249679.85018 & 0.8 \\
\hline & Zo & 1168903.035 & 2.18 & 1168903.04386 & 21.4 \\
\hline \multirow{3}{*}{ LIMN } & $X_{0}$ & 762717.256 & 17.49 & 762717.32546 & -4.5 \\
\hline & $\mathrm{Yo}_{0}$ & -6235556.524 & 8.2 & -6235556.49120 & 2.8 \\
\hline & Zo & 1099500.424 & 4.81 & 1099500.44331 & 10.0 \\
\hline \multirow{3}{*}{ NEIL } & $X_{0}$ & 774610.248 & 21.56 & 774610.33469 & 3.9 \\
\hline & $Y_{0}$ & -6258471.572 & 10.54 & -6258471.52975 & -6.1 \\
\hline & Zo & 952302.985 & 22.41 & 952303.07416 & -1.3 \\
\hline \multirow{3}{*}{ NICY } & $X_{0}$ & 497630.829 & -4.93 & 497630.80884 & 28.3 \\
\hline & $Y_{0}$ & -6259516.912 & -1.78 & -6259516.91892 & -0.2 \\
\hline & Zo & 1115789.529 & -5.79 & 1115789.50632 & 37.0 \\
\hline \multirow{3}{*}{ PUNT } & $X_{0}$ & 565870.292 & 4.43 & 565870.31018 & 15.5 \\
\hline & $Y_{0}$ & -6256745.203 & 1.01 & -6256745.19923 & -1.4 \\
\hline & Zo & 1098060.879 & 6.04 & 1098060.90346 & -74.8 \\
\hline \multirow{3}{*}{ RIDC } & $X_{0}$ & 651566.569 & 7.47 & 651566.59917 & 9.4 \\
\hline & $Y_{0}$ & -6250735.963 & 3.18 & -6250735.95008 & 0.7 \\
\hline & Zo & 1091707.726 & 15.96 & 1091707.78982 & 2.6 \\
\hline \multirow{3}{*}{ SAGE } & $X_{0}$ & 690230.786 & 20.36 & 690230.86744 & 6.8 \\
\hline & $Y_{0}$ & -6256292.416 & 8.15 & -6256292.38308 & -4.5 \\
\hline & Zo & 1032020.601 & 22.27 & 1032020.68962 & 2.5 \\
\hline
\end{tabular}




\section{ANÁLISIS DE RESULTADOS}

Las series de datos se trabajaron en diferencias de coordenadas geocéntricas cuyos valores promedio y de variablidad demostraron que para la coordenada $\mathrm{X}$, el promedio de las diferencias estuvo entre $1,71 \mathrm{~mm}$ (LIBE) y 26,0 mm (SAGE). Para la coordenada $\mathrm{Y}$, las diferencias extremas fueron de $-2,20 \mathrm{~mm}$ (AACR) y 20,49 mm (NEIL) y finalmente para la coordenada $Z$, los valores fueron de 2,83 mm (LIMN) y 26,69 mm (SAGE). Igualmente, la dispersión de las diferencias fue entre $8 \mathrm{~mm}$ y $15 \mathrm{~mm}$ en la coordenada $X$, entre $9 \mathrm{~mm}$ y $16 \mathrm{~mm}$ en la coordenada $Y$ y en la coordenada $Z$ entre $7 \mathrm{~mm}$ y $17 \mathrm{~mm}$ como valores promedios.

En cuanto al error del valor ajustado el promedio para la coordenada $X$ estuvo entre 0,19 $\mathrm{mm}$ y $0,56 \mathrm{~mm}$, mientras que para la coordenada $Y$ entre $0,30 \mathrm{~mm}$ a $0,57 \mathrm{~mm}$ y para la coordenada $Z$, los valores estuvieron entre $0,30 \mathrm{~mm}$ a $0,59 \mathrm{~mm}$. Los límites inferior y superior de los intervalos de confianza para los promedio de diferencias de coordenadas geocéntricas se presentan en la tabla 3 , y se nota como en promedio el rango estuvo entre $1 \mathrm{~mm}$ y $3 \mathrm{~mm}$, representando intervalos pequeños debido a la gran cantidad de datos y garantizando en principio que eventuales observaciones atípicas sean fáciles de detectar.

En cuanto a la aplicación del test de errores groseros mediante la rutina VelMIC, los resultados demostraron que para:

- Estación AACR, se tuvo un total de una sola observación grosera en la coordenada $X$, la cual presentó un residuo de casi $20 \mathrm{~mm}$. En el caso de las coordenadas $Y$ y $Z$ no se detectaron observaciones groseras según los resultados del test y se estimó que los valores originales de coordenadas deberían asumirse con un error inicial de $5 \mathrm{~mm}$ en la coordenada $X, 14 \mathrm{~mm}$ en la coordenada $\mathrm{Y}$ y $4 \mathrm{~mm}$ en la coordenada $Z$.

- Estación CRLP, se tuvo una sola observación grosera en la coordenada X con un residuo de unos $-19 \mathrm{~mm}$. No hubo errores groseros en las otras dos coordenadas y la estimación de errores iniciales para las observaciones de esta estación son de $5 \mathrm{~mm}$ en la coordenada $X, 8 \mathrm{~mm}$ y $4 \mathrm{~mm}$ en la coordenada $Z$.

- Estación ETCG presentó dos observaciones marcadas como groseras en la coordenada $Y$ con residuos de $-37 \mathrm{~mm}$ y $45 \mathrm{~mm}$. Las observaciones correspondientes a las coordenadas $X$ y $Z$ no presentaron errores groseros y la estimación de errores iniciales fue de $4 \mathrm{~mm}$ en la coordenada $X, 8 \mathrm{~mm}$ en la coordenada $Y$ y $4 \mathrm{~mm}$ en la coordenada $Z$.

- Estación LIBE presentó 4 observaciones groseras según el test. Una observación en el vector de coordenadas $X$, una en el vector de coordenadas $Y$ y dos en el vector de las observaciones para $Z$. Los residuos fueron respectivamente en promedio de $25 \mathrm{~mm},-40 \mathrm{~mm},-16 \mathrm{~mm}$ y $-18 \mathrm{~mm}$. Posteriormente la estimación de errores iniciales para las observaciones brindó resultados de $5 \mathrm{~mm}$ en la coordenda $X$, de $9 \mathrm{~mm}$ en la coordenada $Y$ y de $4 \mathrm{~mm}$ en la coordenada $Z$.

- Estación LIMN presentó un total de 4 observaciones groseras en la coordenada X con residuos promedio de $24 \mathrm{~mm},-28 \mathrm{~mm},-26 \mathrm{~mm},-25 \mathrm{~mm}$, de 4 en la coordenada Y con valores de $48 \mathrm{~mm}, 51 \mathrm{~mm},-43 \mathrm{~mm}, 42 \mathrm{~mm}$ y dos observaciones en la coordenada $Z$ con residuos de $-19 \mathrm{~mm}$ y $-19 \mathrm{~mm}$. La estimación de errores inciales para las observaciones de esta estación fueron de $5 \mathrm{~mm}$ en la coordenada $X$, de $10 \mathrm{~mm}$ en la coordenada $Y$ y de $4 \mathrm{~mm}$ en la coordenada $Z$.

- Estación NEIL tuvo un total de 3 observaciones groseras, una en cada coordenada con valores de residuo de $41 \mathrm{~mm},-35 \mathrm{~mm}$ y $41 \mathrm{~mm}$ respectivamente para las coordenadas $X, Y, Z$. Los errores inciales estimados fueron de $5 \mathrm{~mm}$ en la coordenada $X, 8 \mathrm{~mm}$ en la coordenada $Y$ y $4 \mathrm{~mm}$ en la coordenada $Z$. 
- Estación NICY no presentó errores groseros en los vectores de observaciones según los resultados del test. La posterior estimación de errores iniciales fue de 4 $\mathrm{mm}, 8 \mathrm{~mm}$ y $4 \mathrm{~mm}$ para las coordenadas $X, Y$ y $Z$

- Estación PUNT presentó tres errores groseros según los resultados del test, uno en cada coordenada con residuos de $-48 \mathrm{~mm}, 37 \mathrm{~mm}$ y $-15 \mathrm{~mm}$ respectivamente. Los errores iniciales estimados fueron de $5 \mathrm{~mm}$ en la coordenada $X$, de $8 \mathrm{~mm}$ en la coordenada $Y$ y $4 \mathrm{~mm}$ en la coordenada $Z$ respectivamente.

- Estación RIDC presentó un total de 3 observaciones groseras en la coordenada $X$ con residuos de $20 \mathrm{~mm},-24 \mathrm{~mm}$ y $-19 \mathrm{~mm}$. En la coordenada $Y$ se detectaron 2 observaciones groseras con un residuos de $41 \mathrm{~mm}$ y $39 \mathrm{~mm}$. La coordenada Z no presentó observaciones groseras. La estimación de errores inciales fue de $5 \mathrm{~mm}$ en la coordenada $X$, de $9 \mathrm{~mm}$ en la coordenada $Y$ y de $4 \mathrm{~mm}$ en la coordenada $Z$.

- Estación SAGE solamente presentó una observación grosera con un residuo de $42 \mathrm{~mm}$ detectado en la coordenada X; las otras dos coordenadas no presentaron errores groseras según los resultados del test y la estimación inicial de los errores para las observaciones fue de $5 \mathrm{~mm}$ para las observaciones en la coordenada $X$, de $9 \mathrm{~mm}$ para las observaciones de la coordenada $Y$ y de $4 \mathrm{~mm}$ en las observaciones de la coordenada $Z$.

En total, luego de los ajustes parciales se detectaron un total de 35 observaciones groseras cuyos residuos promediales estuvieron entre $-40 \mathrm{~mm}$ y $40 \mathrm{~mm}$. La coordenada que presentó mayor número de observaciones groseras fue la $X$ con un total de 15 observaciones; mientas que las coordenadas $Y$ y $Z$ presentaron un total de 13 y 7 observaciones groseras respectivamente.

La validación de los velocidades resultantes derivadas de los datos procesados por PPP (ver tabla 5) se hizo por medio de la solución SIRGAS SIR15P01, reducidos a la época de referencia 2013,0. Se usaron las velocidades y las coordenadas de referencia de esta solución y se extrapolaron a la época 2017,0 . Los resultados de esta comparación se presentan en tabla 6 en el cual se muestra un valor de diferencia para la coordenada $X$ entre $-6,0 \mathrm{~mm}$ y $28 \mathrm{~mm}$. Para la coordenada $Y$ se tuvo valores de diferencias entre - 6 $\mathrm{mm}$ y $8 \mathrm{~mm}$ y para la coordenada $Z$ entre $-75 \mathrm{~mm}$ y $37 \mathrm{~mm}$. De los valores anteriores se determinaron las diferencias promedio en las coordenadas los cuales son de $11 \mathrm{~mm}$ para la coordenada $X$, de $1 \mathrm{~mm}$ en la coordenada $Y$ y de $-19 \mathrm{~mm}$ en la coordenada $Z$, lo cual representa un excelente consistencia de la solución de PPP respecto a la solución SIR15P01. Se encontró una consistencia en las coordenadas $X$ y $Z$ de unos $15 \mathrm{~mm}$ en valor absoluto y de prácticamente nula en la coordenada $Y$ la cual corresponde a la altura en un sistema topocéntrico. Esto puede deberse a que esta coordenada presenta generalmente una mayor variabilidad en los datos.

El test de errores groseros programado en lenguaje de MatLab, quizás es una primera buena aproximación para la detección de valores atípicos en los conjuntos de datos ya que realiza la comparación sobre los residuos de las observaciones los cuales siguen el principo de mínimos cuadrados y se calculan con base en los datos y los pesos de las observaciones, por lo que este residuo representa un mejor parámetro para la comparación en lugar del dato inicial. Sin embargo, a pesar de que el test de errores groseros implementado en este estudio logró detectar algunos datos atípicos, éstos no constituyen realmente una diferencia significativa en el cálculo final de las velocidades, debido a la gran cantidad de observaciones originales.

\section{Referencias}

Alves Costa, S., Silva, A. D., Moura, N. J. D., Gende, M., y Brunini, C. (2009). Differential and precise point positioning in the south american region with ionosphere maps.observing our changing earth. International Association of Geodesy Symposia 133. 
Kuang, S. (1996). Tgeodetic network analysis and optimal design. Estados Unidos de América. ISBN 0-9668162-0-X.: Concepts and Applications.

Mireault, Y., Tétreault, P., Lahaye, F., Hëroux, P., y Kouba, J. (2008). Online precise point positioning. a new, timely service from natural resources canada. GPS World.

Moya, J., Núñez, C., y Cubero, J. (2017). Usando la red de estaciones sirgas de costa rica para la cuantificación de las discrepancias respecto de un procesamiento ppp en línea. Revista de Ingeniería. Universidad de Costa Rica. 39-55, ISSN: 2215-2652.

Moya, s., J.and Bastos, y Rivas, M. J. (2014). Cálculo, mediante la aplicación del algoritmo de ajuste por mínimos cuadrados, de los componentes de velocidad para estaciones gnss

continuas. Revista UNICIENCIA. Universidad Nacional.
Niemeier, W. (2002). Ausgleichungsrechnung. Alemania: Walter De Gruyter.

Pelzer, H. (1985). Geodätische netze in lands-und ingenieurvermessung ii. Hannover, Alemania.

Perović, G. (2005). Least squares. ISBN: 86-907409-0-2. Belgrado, Serbia: University of Belgrade, Faculty of Civil Engineering, Belgrade.

SIRGAS. (2018). Sistema de referencia geocéntrico para las américas. Autor. Descargado de http://www.sirgas.org 